\title{
Saïd et Genet. La représentation des Palestiniens et la question de l'orientalisme chez Jean Genet
}

\author{
Karl Ågerup (University of Gothenburg, Sweden)
}

\begin{abstract}
During the last two decades of his life, Jean Genet (1910-86) stopped writing novels and plays. Instead he wrote non-fictional stories and essays, many of with depicted Palestinian soldiers and refugees living in Jordan and Lebanon. In this article, Genet's representation of Palestinians is discussed in the perspective of Edward Said's orientalism theory. At first sight, the fact that Genet is a Westerner writing in French about a foreign people whose language he does not speak might suggest that he moulds Palestinian reality in order to fit Western thought and Western aesthetics, thereby producing orientalist discourse. However, rather than exploiting the East to strengthen Western identity, Genet uses Eastern reality to undermine Western thought. It is concluded that Genet does not meet Said's criteria of orientalism since the Palestinians are situated at the centre of his world and occupy a privileged position that surpasses the interests of Western politics, culture, and identity.
\end{abstract}

Keywords: orientalism, Palestinians, Genet, Western, identity

\section{Introduction}

Après une période de silence et de dépression, Jean Genet (1910-86) reprend la parole dans les années 1970 en s'engageant pour plusieurs groupes révolutionnaires, dont la résistance palestinienne. Jusqu'à sa mort en 1986, Genet fait de nombreux voyages en Jordanie et au Liban pour vivre avec des feddayin et des réfugiés palestiniens. Entre-temps, il écrit et fait publier cinq textes sur les Palestiniens, dont les plus connus sont le témoignage «Quatre heures à Chatila » et le livre de souvenirs Un captif amoureux. Étant donné que Genet - un écrivain blanc fasciné par le monde arabe - se donne pour tâche primaire pendant cette époque de décrire le Proche orient et d'expliquer comment vivent et pensent les Palestiniens il paraît raisonnable d'étudier son œuvre à la lumière de la théorie de l'orientalisme.

Edward W. Saïd développe dans son œuvre Orientalisme - l'Orient créé par l'Occident (originairement publié en 1978 sous le titre Orientalism - Western conceptions of the Orient) la thèse que l'Occident a fait usage de l'Orient pour construire son identité et que la représentation occidentale de l'Orient en dit plus sur l'Occident que sur l'Orient. Par une analyse critique des textes écrits par des Occidentaux sur les Orientaux et sur l'Orient, Saïd relève la dimension politique et instrumentale de ce discours occidental portant sur un Orient mythique, un discours donc « orientaliste ». Les représentations que s'est fait l'Occident de l'Orient sont, selon Saïd, le résultat de processus conscients et inconscients destinés à réduire 
l'Orient à une matière exotique qu'il faut domestiquer (Saïd 1978). Le terme « discours» s'entend ici dans la tradition de Michel Foucault qui, dans L'ordre du discours, a supposé que

dans toute société la production du discours est à la fois contrôlée, sélectionnée, organisée et redistribuée par un certain nombre de procédures qui ont pour rôle d'en conjurer les pouvoirs et les dangers, d'en maitriser l'évènement aléatoire, d'en esquiver la lourde, la redoutable matérialité. (Foucault 1971, 10-11)

De même, l'hypothèse foucaldienne sur la relation entre le savoir et le pouvoir avancée dans Archéologie du savoir (Foucault 1969) est centrale pour Saïd, qui part du principe que le « savoir » que l'Occident s'est construit sur l'Orient est le résultat d'un exercice de pouvoir plus ou moins automatique et souvent imperceptible. Même si la pensée occidentale sur l'Orient comme entité culturelle et géographique s'est modernisée pendant la période de la décolonisation, la représentation occidentale de l'Arabe reste essentiellement la même. Selon Saïd, l'Occident peint les Arabes comme des êtres agités, parasitaires, violents, traîtres, dangereux et obsédés par le sexe (Saïd 1978, 286-288).

En somme, Saïd identifie dans Orientalisme quatre dogmes orientalistes fondamentaux. Saïd note tout d'abord la notion d'une différence absolue et systématique entre l'Occident et l'Orient. Si l'Occident est rationnel, développé, humain et supérieur, l'Orient est brut et inférieur. Le deuxième dogme est la primauté dans le discours orientaliste des abstractions occidentales sur les preuves concrètes de la réalité orientale. Dans cette logique, la réalité orientale doit être traduite en une langue et une structure occidentales pour prendre une forme réelle et compréhensible. Le troisième dogme se résume à ce que l'Orient est immuable, homogène et incapable de se définir lui-même. Par là même un vocabulaire généralisé occidental, plutôt que l'expression autochtone, est autorisé et considéré dans l'Occident comme scientifiquement objective. Quatrièmement, l'Orient est aux yeux de l'Occident quelque chose qu'il faut craindre ou contrôler par des actes de pacification, par la recherche ou par l'occupation militaire si nécessaire (Saïd 1978, 300-301).

Ensuite, Saïd a développé davantage sa théorie dans Culture and Imperialism, où il met l'accent sur les romans anglais des $18^{\mathrm{e}}$ et $19^{\mathrm{e}}$ siècles (Saïd 1993). Les œuvres littéraires de l'époque coloniale, d'après Saïd, s'arrogent le privilège occidental de décrire le monde et sont ainsi liés à l'impérialisme. En somme, la théorie de l'orientalisme est largement centrée sur la relation entre langue et pouvoir: elle s'occupe des questions de savoir qui a droit à la parole, qui parle pour qui, et pour servir quel intérêt. La thèse principale de Saïd se résume à ce que l'Occident, depuis le colonialisme et jusqu'à nos jours, s'est chargé de définir l'Orient selon des modalités occidentales qui renforcent la position et l'identité superposées occidentales.

Edward Saïd précise dans un article publié dans La revue des études palestiniennes que l'intérêt qu'exprimait Jean Genet pour les Palestiniens n'est pas à considérer comme une pratique orientaliste. Se référant aux fameuses déclarations de Genet sur la charge érotique du monde arabe (Genet 1991a, 156), Saïd affirme que rien ne laisse penser que Genet cherchait une position paternelle et bienveillante auprès des Palestiniens :

On n'a aucune preuve d'un usage chez lui de la connaissance et des sciences coloniales et l'on ne voit aucun recours dans ses écrits ni dans ses déclarations à des stéréotypes touchant les coutumes des Arabes, leurs mentalités, un passé tribal mythique, tous ces clichés qu'il aurait fort bien pu utiliser pour interpréter ce qu'il voyait et ressentait. Quelle que soit la manière dont il fit contact avec les Arabes (Un captif amoureux laisse penser 
qu'il fut pour la première fois amoureux d'un Arabe lorsqu'il se trouvait, jeune soldat de dix-huit ans, à Damas, il y a de cela un demi-siècle), il pénétra dans cet espace et il y vécut, non pas comme explorateur d'exotisme, mais comme un homme aux yeux de qui les Arabes avaient une actualité réelle, une présence à laquelle il trouvait du plaisir et auprès de laquelle il se sentait à son aise, même s'il était lui-même différent, et le demeurait. Dans le contexte général d'un orientalisme dominant, qui commandait, codifiait, articulait virtuellement toute la connaissance et l'expérience occidentales touchant le monde arabo-islamique, il y a quelque chose de subversif - subversion tranquille mais héroïque aussi - dans la relation extraordinaire que Genet entretenait avec les Arabes. (Saïd 1991, 97)

Plutôt que de condamner Genet, déclare Saïd, les Arabes se doivent de le lire consciencieusement même s'ils n'ont pas l'habitude de rencontrer un Occidental qui les estime. Saïd sépare Genet de T.E. Lawrence. Si ce dernier, orientaliste selon Saïd, faisait preuve d'un « esprit d'affirmation » et « goût naturel pour le pouvoir froidement exercé », Genet exprimait plutôt " l'érotisme et la soumission authentique à l'entraînement d'un engagement politique et passionné à la fois » (Saïd 1991, 100). En conclusion, Saïd voit l'engagement de Genet pour les Palestiniens comme « un acte de solidarité vécue », et une «identification volontaire et amoureuse avec d'autres identités » (101). Le mot-clé sur lequel Saïd insiste dans la conclusion, est celui de « vécu ». Puisque Genet a vécu si longtemps et si intensivement avec les Palestiniens, il sait rendre leur réalité sans la modeler sous une forme occidentale. Certes, le désir érotique qu'éprouve Genet pour le monde arabe n'est pas sans rappeler les conquêtes sexuelles colonialistes. Saïd, cependant, en fait un mérite : l'attitude passionnée de Genet prouve que son amour pour le monde arabe est authentique.

Dans ce cadre théorique et historique, nous nous proposons d'examiner l'écriture de Genet au sujet des Palestiniens après 1970. Edward Saïd, qui était lui-même Palestinien et ami de Genet, avait-il raison d'écarter l'écrivain du discours orientaliste ?

\section{Les Palestiniens chez Jean Genet dans les années 1970}

En tout, Jean Genet a publié cinq textes sur les Palestiniens. Avant d'étudier plus en détail les deux derniers textes, qui sont les plus connus, nous allons évoquer les trois premiers, publiés dans les années 1970. Dans le premier texte, intitulé «Les palestiniens » et publié dans le magazine Zoom en 1971, Genet commente des photographies prises dans un camp palestinien (Genet 2010a). Dans ce texte, Genet décrit par exemple la photo d'un fedayin allongé et armé qui fume sa cigarette en regardant droit dans la caméra. La réflexion de Genet porte sur la symbolique sexuelle de la Kalachnikov et le regard ironique du soldat. Nous avons affaire à un commentaire formulé par un écrivain occidental à partir d'une photo prise par un autre Occidental. Loin du Palestinien qui fume sa cigarette sur la photo, Genet explique aux lecteurs occidentaux comment vivent et pensent les fedayin (Genet 2010a, 6869). Le deuxième texte où Genet évoque les Palestiniens est nommé «Les femmes de Djebel Hussein » et fut publié dans Le monde diplomatique en 1974 (Genet 2010b). Le texte est un bref reportage littéraire dans lequel Genet prend une tasse de thé avec quatre femmes palestiniennes dans la périphérie d'Amman. Les femmes parlent de leurs fils qui sont partis faire la guerre. Dans un style neutre, sans explications pédagogiques, Genet se place au plus près de la réalité palestinienne. L'impression de proximité et d'authenticité est renforcée par le fait que ce milieu à l'écart de la guerre est rarement représenté par les médias occidentaux. Les femmes de Djebel Hussein ne sont ni dangereuses ni incontrôlables mais elles sont 
paisibles et calmes autour de leur feu et leur théière. Elles ne sont pas représentées en opposition avec les femmes occidentales mais plutôt en opposition avec leurs fils rebelles dont elles se moquent subtilement. Comme le note El Omari, les abstractions politiques s'absentent :

L'important n'est pas de dire la vie dans les camps ni de décrire la politique jordanienne (des événements qui ancrent l'histoire dans une date précise) mais de dessiner, outre la joie et la gaieté, une théière en aluminium cabossée au milieu d'une maison de trois pierres noircies; de faire voir l'index aigu de la « main très sèche et très belle » montrant les morts. Ces femmes, dont le nom sera «tragédiennes » dans un autre texte de Genet envahissent la scène du réel et créent l'histoire à leur façon. (El Omari 2001, 135)

Genet, remarquablement sensible aux gestes et paroles des Palestiniennes, cherche à rendre le milieu sans faire d'abstractions ni prendre une position supérieure. Encore faut-il retenir qu'il s'agit d'un écrivain occidental qui se rend dans un milieu oriental pour donner, dans une langue et un style occidentaux, son propre compte de leur réalité.

Le troisième texte, intitulé «Près d'Ajloun ", apparaît en 1977 dans une œuvre collective publiée en mémoire du leader palestinien Waël Zwaiter, qui venait d'être assassiné à Rome (Genet 2010c, 137-146). Ce texte est plus abstrait que les précédents ; Genet y réfléchit sur l'identité des fedayin palestiniens et sur le sens de l'arabité, un terme mal compris par les Occidentaux à son sens. Genet se demande quel serait l'équivalent européen de l'arabité, la latinité ? "Capté de l'extérieur, le mot n'est pas vécu, donc pas compris » (139). Ainsi l'écrivain met l'accent sur son expérience directe du monde arabe: pour comprendre les Arabes il faut vivre avec eux, comme lui l'avait fait. Genet déclare dans " Près d'Ajloun » que les fedayin font des rêves bourgeois qui s'opposent à leur idéologie révolutionnaire: ils rêvent de femmes, de mères, de situations tranquilles. L'écrivain, plutôt que de décrire les Arabes comme différents, met donc l'accent sur la similitude entre les jeunes occidentaux et orientaux (141-142). Dans le contexte de l'orientalisme il est cependant intéressant de remarquer que Genet compare les feddayin palestiniens et les soldats bédouins en ce qui concerne leurs manières de danser :

La danse est révélatrice. Au camp de Baga, en Jordanie, j’ai assisté à une fête de réconciliation entre feddayin palestiniens et soldats bédouins. Embarrassés par leur corps et les pas de danse, les Palestiniens étaient lourds, maladroits, presque ridicules, quand les Bédouins étaient admirables. Dans les deux camps la danse était la même, mais si les Palestiniens la considéreraient comme un amusement, pour les Bédouins c'était une parade sacrée, les rassurant sur leurs condition d'hommes dansant pour eux-mêmes, dansant devant Dieu, et dansant pour les femmes. (144)

De même, Genet affirme que « la bravoure guerrière des Bédouins est très grande mais elle n'est rien devant le courage moral des Palestiniens » (145). Enfin, Genet soulève le silence et le calme des femmes palestiniennes. Ainsi, dans « Près d'Ajloun », Genet articule les spécificités des différents groupes arabes qui sont alors comparés entre eux, plutôt que d'être comparés aux Occidentaux.

\section{Genet à Chatila}

Après une nouvelle période de silence et de problèmes de santé, Genet publie en 1982 un texte intitulé «Quatre heures à Chatila » (Genet 2010d), dans lequel il décrit et commente 
le massacre de Chatila au Liban, à savoir le massacre de plusieurs centaines de réfugiés palestiniens par une milice du parti des Phalanges. L'accent mis dans l'historiographie occidentale sur le fait que Genet fut l'un des premiers Occidentaux à entrer dans le camp après le massacre implique, dans l'optique de Saïd, que l'événement ne fut pas réel avant qu'un Occidental le décrive. En effet le discours occidental aurait, selon la théorie de Saïd, tendance à répondre à cette violence par des mesures de contrôle : des campagnes humanitaires, des recherches sociales, des mesures politiques et militaires. Cependant, «Quatre heures à Chatila » est avant tout un compte rendu subjectif et sensuel de cette catastrophe humanitaire. Genet affirme que les ruelles de Chatila sont tellement étroites que le corps d'un enfant palestinien peut les bloquer - une formulation qui évoque l'image d'un auteur qui, à son âge finissant, enjambe des cadavres d'enfants. L'odeur, " familière aux vieillards », ne le gêne pas (Genet 2010d, 177). Genet s'intègre dans le milieu à tel point que les mouches, qui semblent d'abord gênées par lui, finissent par s'habituer à sa présence (184). La narration de Genet se distingue de celle du journaliste anglais Robert Fisk, qui était aussi sur place à Chatila juste après le massacre. L'exposé de Fisk répond aux questions journalistiques de base consistant à savoir «quoi », « quand » et « où », avant de passer aux questions de savoir «comment »et «pourquoi ». De même, Fisk affirme qu'il a dû prendre une longue douche après avoir visité le camp (Fisk 2001, 359). Genet, plutôt que de suivre ce schéma journalistique occidental, reste longtemps parmi les cadavres, visiblement fasciné, voire passionné, avant de contextualiser ses expériences. Si abstraction il y a, elle prend un air étonnamment subjectif. Genet s'approche des morts, il les regarde soigneusement et tente de rendre directement ses impressions.

Si l'on regarde attentivement un mort, il se passe un phénomène curieux : l'absence de vie dans ce corps équivaut à une absence totale du corps ou plutôt à son recul ininterrompu. Même si on s'en approche, croit-on, on ne le touchera jamais. Cela si on le contemple. Mais un geste fait en sa direction, qu'on se baisse près de lui, qu'on déplace un bras, un doigt, il est soudain très présent et presque amical. (Genet 2010d, 178)

Plutôt que d'expliquer le massacre sur une toile de fond occidentale, Genet se tient au plus près de la matière brute en évoquant non seulement les réfugiés assassinés du camp mais aussi les mouches. Lorsqu'il aborde le contexte politique du massacre, Genet ne cherche ni à prendre la position neutre d'observateur ni à superposer la voix du « sage occidental ». À la place, il avoue brutalement qu'il aime les Palestiniens et hait les Israéliens dont l'armée avait, selon lui, facilité le massacre (Genet 2010d:186). Selon Genet, on choisit son camp en fonction d'émotions et d'attractions. Pour lui, les jugements sont irrationnels:

Le choix qu'on fait d'une communauté privilégiée, en dehors de la naissance alors que l'appartenance à ce peuple est native, ce choix s'opère par la grâce d'une adhésion non raisonnée, non que la justice n'ait pas part, mais cette justice et toute la défense de cette communauté se font en vertu d'un attrait sentimental, peut-être même sensible, sensuel ; je suis français, mais entièrement, sans jugement, je défends les Palestiniens. Ils ont le droit pour eux puisque je les aime. Mais les aimerais-je si l'injustice n'en faisait pas un peuple vagabond ?(190)

Edward Saïd, dans le plaidoyer pour Genet que nous avons cité plus haut, met l'accent sur l'amour de Genet pour les Arabes - un amour vécu sur place. Tandis que l'écrivain orientaliste type repart en Occident pour exploiter son amour pour l'Orient et pour soumettre les Arabes à une forme occidentale et les présenter sur une scène occidentale, Genet reste 
autant que possible chez les Palestiniens. Le jugement de Saïd se base ainsi sur une hiérarchie d'amours selon laquelle un amour exprimé sur place vaut plus qu'un amour prononcé de loin (Saïd 1991, 100).

\section{Genet, captif amoureux}

Le cinquième et dernier texte que Genet consacre aux Palestiniens est un livre de 600 pages intitulé Un captif amoureux. Genet travaillait sur ce livre de souvenirs pendant une dizaine d'années; on y reconnaît des scènes et idées que l'auteur avait déjà représentées dans les articles susmentionnés. Par exemple, Genet répète que les fedayin jouent aux cartes avec des cartes imaginaires, une habitude qu'il avait déjà décrite dans «Les Palestiniens » (Genet 2010a, 68). De nouveau, Genet s'intègre dans le milieu révolutionnaire et le représente par une rhétorique personnelle. Il décrit les habitudes quotidiennes sans articuler en quoi elles sont différentes des coutumes occidentales et sans se servir de méthodes explicitement occidentales. Comme dans "Près d'Ajloun ", Genet s'attarde sur des phénomènes reconnaissables au lecteur occidental tels que la Voie lactée (Genet 1986, 17) et la constellation de la Petite Ourse (19). De même, Genet découvre la liberté des femmes palestiniennes, qui se permettent de le fréquenter bien qu'elles soient mariées (12-13). Le fait que Genet vive avec les Palestiniens rend crédible la narration ; lorsque Genet affirme qu'aucun feddai ne se sépare jamais de son fusil, il est facile de le croire puisqu'il ajoute des réflexions précises et des observations apparemment faites sur place :

Aucun des feddayin n'avait lâché son fusil, soit qu'il restât à l'épaule, avec sa courroie en
bandoulière, ou que le feddai le tînt horizontal sur ses genoux, vertical entre eux sans se
douter que cette pose en elle-même était une menace érotique ou mortelle, ou les deux.
[...] Qu'il fit la cuisine, secouât ses couvertures, lût ses lettres, l'arme était presque plus
vivante que le soldat. Au point que je me demande si la fermière voyant venir à elle des
enfants sans armes ne fût pas rentrée chez elle, outragée par la vue d'adolescents nus.
(Genet 1986, 122-123)

Comme le note El Omari, Genet communique par un style également détaillé le rêve que font les Palestiniens de leur propre nation, un rêve tragique, une « réalité-fiction ». Pour inscrire cette absence tragique, prétend El Omari, « il faut d'abord la voir, puis la connaître et enfin la sentir. Voir, connaître, sentir s'entrelacent dans la même écriture non pas seulement pour raconter un épisode de la vie de l'auteur mais surtout pour faire entendre la voix d'un peuple et celle de sa terre » (El Omari 2001, 130-131).

Dans Un captif amoureux, c'est souvent l'Orient qui occupe la place centrale, tandis que l'Occident apparaît comme périphérique. Genet précise dans le livre qu'il ignore l'emplacement géographique de Verdun (Genet 1986, 158) et qu'en Jordanie, il est soumis aux Palestiniens, il est « le Français » qu'ils décident de garder ou rejeter (163). Son discours s'écarte à cet égard du discours orientaliste qui exprime la suprématie occidentale.

Encore faut-il se demander si Genet ne sculpte pas ses héros selon sa propre idéologie. Est-il vrai, par exemple, que les fedayin « ne possédèrent jamais rien » (170), et ne « comptaient même pas leurs morts » (337) ? Si la seconde déclaration ressemble à un mythe de guerre, la première, vraie ou fausse, reflète la situation de Genet qui, lui, ne possédait rien d'autre qu'une petite valise et qui n'avait pas de maison (Ben Jelloun 1977, White 1993). À cela s'ajoute le fait que Genet écrit en français et parle mal l'arabe palestinien. La proximité physique est contrebalancée par cet obstacle linguistique. Toutefois, à la différence des 
écrivains orientalistes désignés par Saïd qui prétendent comprendre et faire comprendre l'Orient à travers des concepts occidentaux, Genet est conscient de la barrière culturelle et linguistique qui le sépare des Palestiniens et il tient compte de cette barrière dans ses raisonnements en soulignant, par exemple, que « les capitales n'existent pas dans l'écriture arabe » (Genet 1986, 177). La représentation du milieu révolutionnaire s'inscrit donc dans un mouvement de va-et-vient entre similitude et différence, par lequel l'auteur expose son amour pour les Palestiniens.

\section{Conclusion}

En gros, la représentation des Palestiniens chez Jean Genet n'apparaît pas comme orientaliste selon la définition d'Edward Saïd. Genet se positionne au plus près des Palestiniens et se présente comme leur égal. Les préjugés orientalistes listés par Saïd sont rares dans les textes étudiés : les Palestiniens représentés par Genet travaillent bien en groupe, sont rationnels et se maîtrisent. En revanche, le mépris de Genet pour l'hypocrisie des hauts fonctionnaires resurgit de ses textes plus anciens, des textes situés en Europe. Ainsi, on peut repérer dans sa représentation du Proche Orient la tendance de Genet à s'allier à ceux qui se situent au plus bas des hiérarchies de pouvoir. Dans l'univers de Genet les différences de comportement et de pensée chez les hommes dépendent plus des conditions sociales que des ethnicités ou origines culturelles. Plutôt que les hommes, ce sont les institutions qui sont barbares ; la violence des opprimées apparaît comme parfaitement logique. Cette pensée, développée par ailleurs avec plus de précision dans son texte sur la RAF intitulé «Violence et brutalité » (Genet 1977), résonne dans les textes sur les Palestiniens, dans lesquels la brutalité démesurée des armées jordanienne et israélienne est comparée à la résistance sensée et justifiée des Palestiniens.

En ce qui concerne le deuxième dogme orientaliste formulé par Saïd, à savoir la primauté de l'abstraction occidentale sur la réalité orientale concrète, l'examen des cinq textes nous a permis de constater que la réalité orientale est remarquablement présente dans l'écriture de Genet, et que les abstractions sont de nature subjective et tâtonnante. Ainsi dans "Quatre heures à Chatila », plutôt que de faire appel aux experts ou de faire des analyses politiques justifiables, Genet fait des observations concrètes dans le camp et culpabilise sans objectivité feinte l'armée israélienne qu'il déclare haïr (Genet 2010d : 186). Quant au troisième dogme orientaliste, à savoir celui qui consiste à présenter l'Orient comme incapable de se définir lui-même, il est certain que le métier de l'écrivain consiste à rendre une version personnelle du monde : l'écrivain se réserve le droit de définir l'autre. Genet, dans les textes étudiés, parle pour les feddayin et couvre ainsi leurs voix de la sienne. On constate tout de même qu'explicitement Genet n'affirme guère que les Palestiniens sont immuables, homogènes, ou incapables de se définir eux-mêmes.

En ce qui concerne le quatrième dogme, plutôt qu'inciter les Occidentaux à craindre les Palestiniens, Genet accentue la menace que constituent les autorités occidentales - non seulement pour les Palestiniens et mais aussi pour les Occidentaux eux-mêmes. Ceux qu'il faut pacifier et contrôler, selon Genet, ne sont pas tant les Palestiniens que les Occidentaux, ou que les milices jordaniennes, phalangistes et israéliennes. Ainsi, la dichotomie OccidentOrient n'est pas toujours utile dans l'analyse de l'écriture de Genet. Même si Genet ne peut pas échapper à son héritage européen, ses textes ne renforcent guère la position surplombante de l'Occident. Jean Genet vise plutôt à remettre en cause cette position. En cela, le dernier 
Genet est politique ; il soutient les Palestiniens, tout comme il avait soutenu plus tôt d'autres groupes révolutionnaires tels que la RAF et les Black Panthers. Jérôme Neutres, dans Genet sur les routes $d u$ sud, distingue l'œuvre de Genet de la littérature engagée, appelant l'écriture de Genet «compromise » plutôt qu'engagée :

L'écriture compromise se distingue de la littérature engagée par un investissement radical de l'écrivain dans une réalité politique. N'est plus seulement engagée une facette de l'écrivain - son opinion politique sur telle ou telle cause - mais tout son être. [...] Alors surgit une expérience irréductible qui demeure bien au-delà de la circonstance historique. [...] On retrouve une compromission de cet ordre chez Genet. (Neutres 2002, 246-247)

L'analyse de Neutres s'harmonise avec celle de Saïd en ce qu'elle souligne la solidarité vécue de Genet, une solidarité plus complète que celle de l'écrivain engagé. Encore faut-il ajouter que Genet s'approche d'une fraction limitée du peuple palestinien, à savoir les fedayin et leurs familles, ainsi que de quelques écrivains et politiciens exilés. C'est cette fraction particulièrement révolutionnaire qui l'intéresse. La révolte est selon Genet ce qu'il y a de plus vivant, plus signifiant et plus beau dans le monde. Ainsi Genet précise-t-il dans le début d'Un captif amoureux que « les Noirs étaient les caractères sur la feuille blanche de l'Amérique » (Genet 1986, 12). Les Black Panthers portent le sens, tandis que les Blancs ne sont qu'un papier vide. Aussi raciste que cette déclaration puisse paraître, l'attention de Genet, plutôt que vers une ethnicité ou une culture, est dirigée vers une certaine pratique, à savoir la pratique de la révolte qui, pour Genet, n'est pas tant militaire qu'esthétique et sémantique. Lorsqu'il évoque la coutume des fedayin de jouer aux cartes sans cartes, par exemple, ce n'est pas seulement pour examiner une habitude micro-culturelle mais surtout pour exprimer une réalité concrète. Tant que faire se peut, Genet s'abstient de considérer de haut la réalité palestinienne ou de la placer dans une structure occidentale. Plutôt qu'ethnologique, sa perspective est phénoménologique, subjective et poétique. Lorsqu'exceptionnellement, il prend une position politique, Genet se positionne toujours du côté des déshérités. L'inégalité de force entre l'Occident et l'Orient est aussi réelle pour Genet que pour Saïd, et les deux, chacun de sa manière, critiquent cette inégalité. Le rôle de Genet dans cette machine critique anticoloniale est de soulever la réalité palestinienne, d'en exposer la matérialité signifiante. Les textes que nous avons examinés démontrent que les dogmes orientalistes sont absents de la réalité palestinienne telle qu'elle a été conçue par Jean Genet. À la différence du discours orientaliste, les Arabes de Genet ne sont ni agités, ni incontrôlables, ni obsédés par le sexe. L'Orient, dans ces textes, est capable de se définir luimême : les fedayin se battent tous les jours pour définir un territoire palestinien aux dépens des états voisins soutenus par les institutions occidentales. De même, plutôt que de comprendre et de faire comprendre les Arabes en se servant de références occidentales, Genet présente le monde arabe tel qu'il le voit et il s'en sert pour critiquer la vision du monde occidentale.

Encore faut-il ajouter que la langue de Genet est forcément occidentale, de même que les plateformes de publication qu'il utilise. Dans l'optique de l'orientalisme, le point le plus important est que Genet place les Palestiniens au centre de son monde, et cela justement parce qu'ils sont en diaspora. Ce qui caractérise les Palestiniens dans l'œuvre de Genet n'est ni leur ethnicité ni leur religion, mais leur position dans la hiérarchie sociale et politique internationale. Ils sont l'équivalent de cet enfant des rues plein de vengeance et d'humour que l'écrivain concevait dans son premier volume autobiographique intitulé Journal du voleur; ce 
qui l'intéresse, plutôt que de décrire l'Orient, c'est le soulèvement réel et pratique de l’opprimé.

\section{Bibliographie}

Ben Jelloun, Tahar. 1977. « Pour Jean Genet ». Le Monde, 24 Sep. 1977.

El Omari, Basma. 2001. «'La dernière image du monde' ou l'écriture de Jean Genet sur les Palestiniens ». Études françaises vol. 37, no. 3, pp. 129-146.

Fisk, Robert. 2001. Pity the Nation. Lebanon at War. Oxford : Oxford UP.

Foucault, Michel. 1969. Archéologie du savoir. Paris : Gallimard.

-- -. 1971. L'ordre du discours. Paris : Gallimard.

Genet, Jean. 1949. Journal du voleur. Paris : Gallimard.

- - . 1977. «Violence et brutalité ». Le Monde, 2 Sep. 1977, p. 1.

-- - 1986. Un captif amoureux. Paris : Gallimard.

- - . 1991a. «Interview avec Hubert Fichte ». In L'ennemi déclaré. Paris : Gallimard.

- - . 2010a. « Les Palestiniens ». In L'ennemi déclaré. Textes et entretiens choisis

1970-1983, pp. 57-76. Paris : Gallimard.

- - - 2010b. «Les femmes de Djebel-Hussein ». In L'ennemi déclaré. Textes et entretiens choisis 1970-1983, pp. 83-86. Paris : Gallimard.

- - - 2010c. « Près d'Ajloun ». In L'ennemi déclaré. Textes et entretiens choisis 1970-1983, pp. 137-146. Paris : Gallimard.

- - . 2010d. «Quatre heures à Chatila ». L'ennemi déclaré. Textes et entretiens choisis 19701983, pp. 175-204. Paris : Gallimard.

- - . 2010e. «Entretien avec Hubert Fichte», L'ennemi déclaré. Textes et entretiens choisis 1970-1986, pp. 87-135. Paris: Gallimard.

Neutres, Jérôme 2002. Genet sur les routes du sud. Paris : Fayard.

Saïd, Edward W. 1978. Orientalism. Western Conceptions of the Orient. Londres : Penguin.

- - - 1991. «Les derniers écrits de Jean Genet», Revue d'études palestiniennes, No. 39, Printemps 1991, pp. 73-86.

- - - 1993. Culture and Imperialism. New York : Vintage.

White, Edmund. 1993. Genet. Londres : Chatto \& Windus. 